\title{
Angular Distribution of Damping Coefficient of Ablated Particle in Pure He, Ne, and Ar Gases
}

\author{
Yinglong Wang, Xiaolin Zhai, Xuecheng Ding, Ziqiang Hu, Lizhi Chu, \\ Zechao Deng, Weihua Liang, Yajun Zhao, and Guangsheng Fu \\ College of Physics Science and Technology, Hebei University, Baoding 071002, Hebei, China \\ Correspondence should be addressed to Yinglong Wang, hdwangyl@hbu.cn
}

Received 5 June 2011; Revised 26 July 2011; Accepted 26 July 2011

Academic Editor: Gong Ru Lin

Copyright (C) 2011 Yinglong Wang et al. This is an open access article distributed under the Creative Commons Attribution License, which permits unrestricted use, distribution, and reproduction in any medium, provided the original work is properly cited.

\begin{abstract}
To investigate the angular distribution of damping coefficient of ablated particle under various ambient gases, nanocrystalline silicon films are systemically deposited on a circular substrate by pulse laser ablation in pure He, Ne, and Ar gases, respectively. Scanning electron microscopy images and Raman and X-ray diffraction spectra indicate that the average size of Si nanoparticles decreases with the increase of the departure angle between the film and the plume, and Ne gas induces the smallest and most uniform Si nanoparticles in size among all the three gases. Further theoretical simulation demonstrates the bigger the departure angle, the smaller the damping coefficient of ablated particle, and the damping coefficient in Ne gas is largest for the same angle, implying the most effective energy transfer between Si and ambient atoms.
\end{abstract}

\section{Introduction}

There is a significant growing interest in nanocrystalline silicon (nc-Si) thin films [1-3] containing a lot of Si nanoparticles (np-Sis) for their potential applications in optoelectronic integration and high-efficiency solar cell [4-7]. In these applications, the controllability of the average size and size distribution of the $\mathrm{np}$-Sis in the prepared films should be crucial aspects to be considered. In order to better control the size and its distribution of the np-Sis, it is fundamental and necessary to study the main factors that affect nucleation and growth during their preparation. Several preparation methods of the nc-Si films have been developed, such as the chemical vapor deposition (CVD) $[8,9]$, magnetron sputtering (MS) [10], molecular-beam epitaxy (MBE) [11, 12], heterogeneous dilution (HD) [13], and pulsed laser deposition (PLD) [14-17]. PLD technology is of great importance due to the high growth rate and high purity of the prepared thin films [18]. For PLD, the inert gas is introduced into the deposition chamber as the ambient gas, and both the experimental and theoretical results demonstrate that the average size of $\mathrm{np}$-Sis can be altered by modulating the type of ambient gas, pressure, and so on. During nucleation and growth of the np-Si, collisions between Si atoms and ambient gas are crucial. An inertia fluid model can consistently explain the experimental results on dependence on the size of np-Sis He pressure [19]. This model indicates that the drag force $(f)$ of the ablated particle is proportional to the square of velocity $(v)$, namely, $f=\alpha v^{2}$, in which $\alpha$ is the damping coefficient. And the kinetic energy difference between ablated particles initially ejected and ablated particles at end of flight is proportional to the cohesive energy of the as-formed np-Sis. Additionally, the size distribution of np-Sis prepared by PLD at various gases ( $\mathrm{He}, \mathrm{Ar}$, and $\mathrm{Ne}$ ) has been studied [20]. The results show that $\mathrm{Ne}$ induces the most uniform np-Sis among all the three deposition gases, which mainly results from the adjacent degree between the ambient gas and $\mathrm{Si}$ atomic weight. However, the above discussions are restricted to the axial direction of plasma.

In this paper, we report the dependence of damping coefficient on angle during PLD in pure $\mathrm{He}, \mathrm{Ne}$, and Ar gases, respectively.

\section{Experimental Setup}

The pulse laser deposition equipment is composed of a laser source and a vacuum system, as shown in Figure 1. 


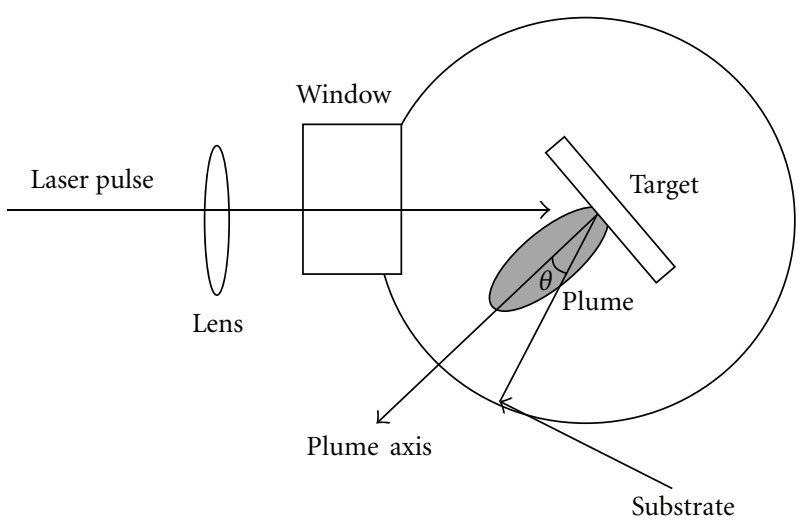

Figure 1: The schematic diagram of the experiment used to study the angular distribution of damping coefficient of ablated particle in pure $\mathrm{He}, \mathrm{Ne}$, and Ar gases.



(a)

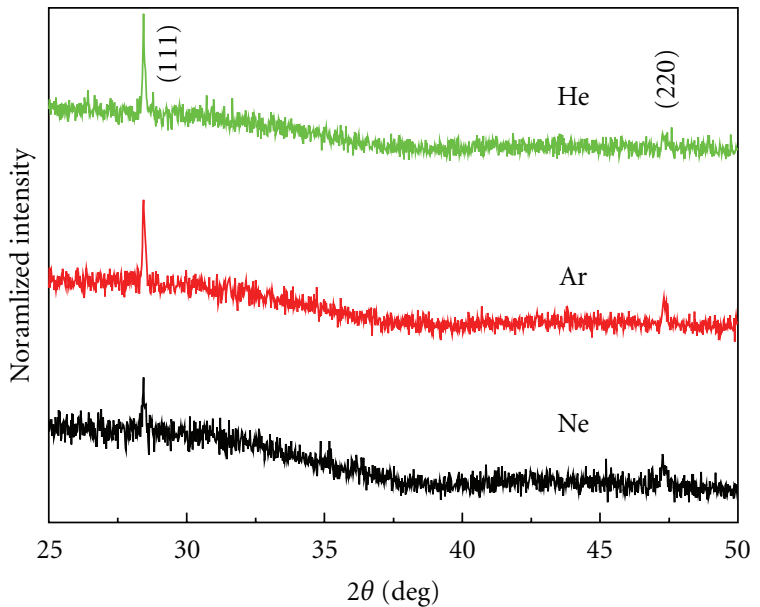

(b)

FIgURE 2: The Raman sepctrum (a) and XRD (b) of Si films deposited by pulsed laser deposition in $10 \mathrm{~Pa} \mathrm{He,} \mathrm{Ne}$, and Ar gases, respectively.

A Lambda Physik XeCl excimer laser (wavelength: $308 \mathrm{~nm}$, pulse duration: $15 \mathrm{~ns}$, repetition: $1 \mathrm{~Hz}$, energy fluence: $4 \mathrm{~J} / \mathrm{cm}^{2}$ ) is focused on surface (ablation spot area: $2 \mathrm{~mm}^{2}$ ) of a single crystalline $\mathrm{Si}$ target (resistivity: $3000 \Omega \cdot \mathrm{cm}$ ) continually rotating during the deposition, intersection angle of the laser and target surface being $45^{\circ}$. A series of single Si (111) or glasses wafer are placed along the inner half circle of the substrate with diameter of $3 \mathrm{~cm}$ and center of circle locates at the ablation spot of the laser. Highpurity $(99.9995 \%) \mathrm{He}, \mathrm{Ne}$, and $\mathrm{Ar}$ are introduced into the chamber, respectively, as the ambient gases are to be kept at $10 \mathrm{~Pa}$ after the base pressure reached $2 \times 10^{-4} \mathrm{~Pa}$. The nc-Si films are directly deposited on the substrate at the room temperature, and deposition time is 3 minutes and 3 hours for the Si and glasses wafer, respectively. The JSM7500 type lull emission scanning electron microscopy (SEM) of Japanese electronics manufacturer, Rigaku D/Max type of X-ray diffraction (XRD) of Japan Rigaku Production Company and Raman scattering of MLI-2000 type apparatus are employed to characterize the surface morphology and microstructure of Si films.

\section{Results and Discussions}

In the experiment, the nc-Si thin films are, respectively, deposited on the glass wafer at the $0^{\circ}$ angle (facing the plume) under $\mathrm{He}, \mathrm{Ne}$, and $\mathrm{Ar}$ and then measured by the Raman spectrum and X-ray diffraction (XRD). The results are shown in Figures 2(a) and 2(b), respectively. In Figure 2(a), the Raman spectrum peaks of the thin films are located at $519.1,518.2$, and $517.4 \mathrm{~cm}^{-1}$, respectively, all of them deviating from the monocrystalline Si peak of $520 \mathrm{~cm}^{-1}$. In Figure 2(b), the $28.4^{\circ}$ and $47.3^{\circ}$ spectra with board width are present in XRD spectrum of the films, which correspond to Si (111) and (220) crystal diffraction, respectively, compared with the standard PDF cards. The measured values above indicate that $\mathrm{np}$-Sis have been formed in the film. 


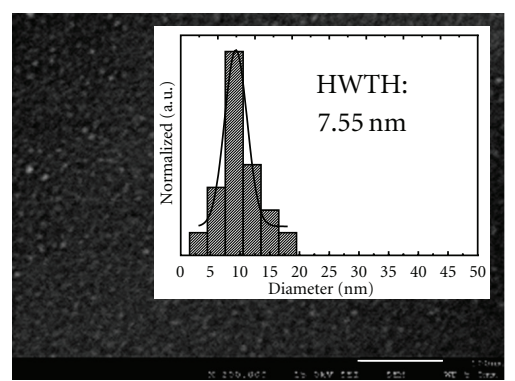

(a)

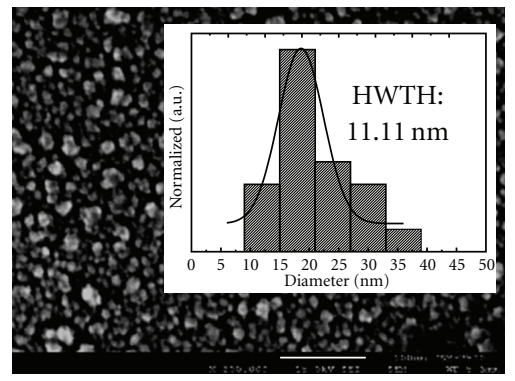

(d)

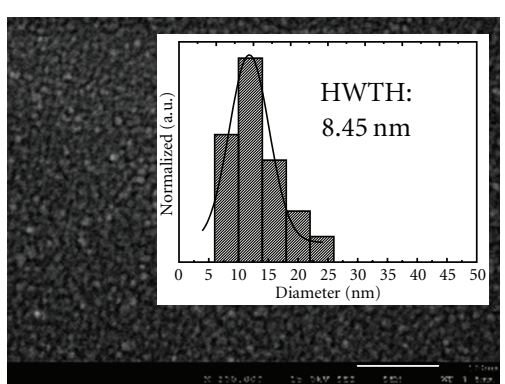

(b)

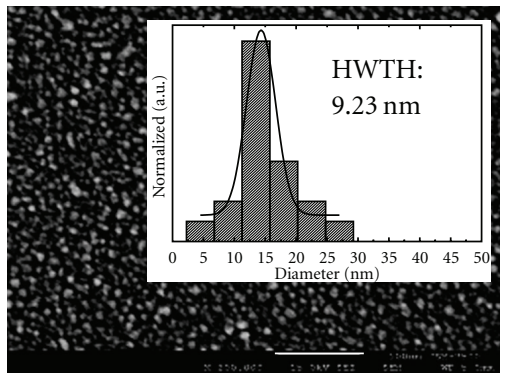

(e)

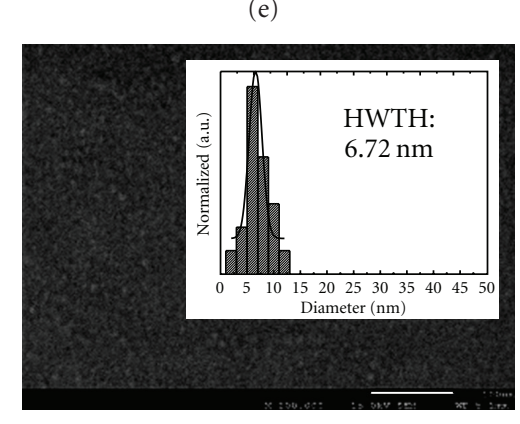

(g)

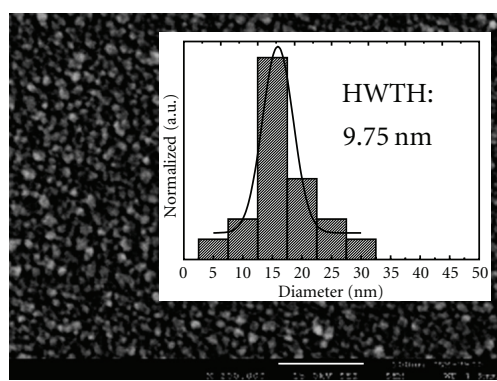

(c)

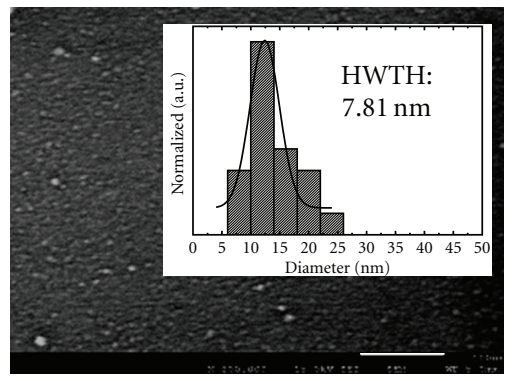

(f)
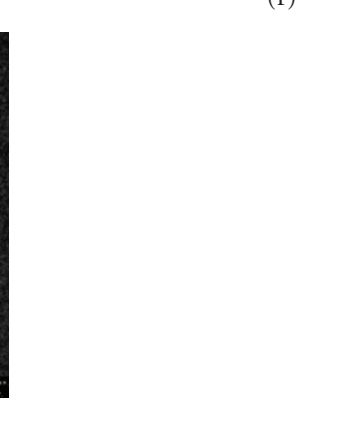

Figure 3: SEM images of the Si films deposited by pulsed laser deposition in 10 Pa He environment gas, where (a), (b), (c),(d), (e), (f), and (g) correspond to various angles of fixed Si wafers: $-80^{\circ},-60^{\circ},-30^{\circ}, 0^{\circ}, 30^{\circ}, 60^{\circ}$, and $80^{\circ}$, respectively.

3.1. The Average Size versus the Angle. The SEM images of the nc-Si films prepared at the angles of $-80^{\circ},-60^{\circ},-30^{\circ}$, $0^{\circ}, 30^{\circ}, 60^{\circ}$, and $80^{\circ}$ under $\mathrm{He}, \mathrm{Ar}$, and $\mathrm{Ne}$, respectively, are present in $(\mathrm{a})-(\mathrm{g})$ of Figures 3,4 , and 5 , in which the insets present the corresponding size distribution of $\mathrm{np}$ Sis. Obviously, the nanoparticles are inserted in the films. Comparing the data at the same angle in the three gases, one can distinguish that the nanoparticles are more uniformly distributed in films prepared in $\mathrm{Ar}$ and $\mathrm{Ne}$ than in $\mathrm{He}$ and the sizes of nanoparticles formed in $\mathrm{Ar}$ and $\mathrm{Ne}$ are smaller than the ones in $\mathrm{He}$ at the $0^{\circ}$ angle. At the same time, the widths of the size distribution of the nanoparticles increase gradually in $\mathrm{Ne}, \mathrm{Ar}$, and $\mathrm{He}$, namely, the probable radius of the nanoparticles increases gradually.

In order to quantitatively study the changing relationship between the size distribution of the nanoparticles and the angles in the three ambient gases, more than 100 nanoparticles in each SEM image are statistically analyzed by Photoshop software, displayed in Figure 6. The results show that the size of np-Sis decreases with the increase of the angle, all for $\mathrm{He}, \mathrm{Ar}$, and Ne. Moreover, the average sizes of nanoparticles in all the films prepared under the three gases are maximal at $0^{\circ}$, namely, at the position facing the plume. Because the substrate is not heated, the nanoparticles are formed by undergoing gas phase nucleation [21]. After the pulse laser ablates the single crystalline Si target, ablation particles are ejected out to all directions, with velocities approximately satisfying the Maxwell velocity distribution [22], and then transported and deposited on the substrates. In the transporting process, the ablated particles collide with the ambient gas atoms and constantly lose kinetic energy which supplies the cohesion energy forming np-Sis [23]. The initial velocity of ablation particles is related to the propagation direction and has the maximal value along axial direction of the plume. For $0^{\circ}$ with maximal velocity, the lost kinetic energy is maximal; thus, the formed $\mathrm{np}-\mathrm{Si}$ is the largest. Because the velocity of the ablated particles decreases with the angles, the smaller nanoparticles will be formed at larger angle. As we know, the adjacent degree between the ambient and $\mathrm{Si}$ atomic weight is the crucial factor to the size and its distribution of np-Sis [24]. If the ambient atomic weight is close to Si atomic weight, the size distribution range 




(a)

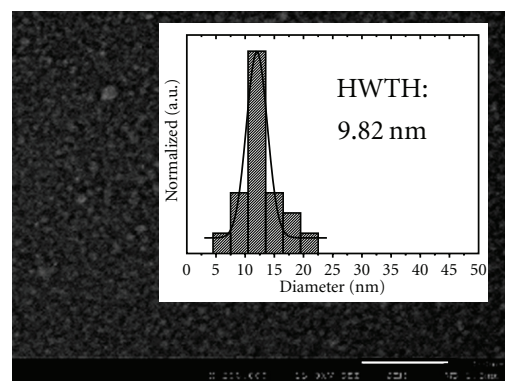

(d)

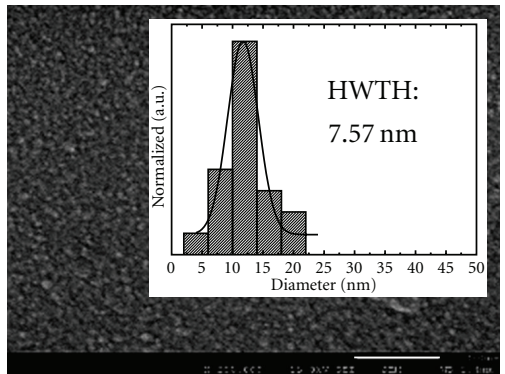

(b)

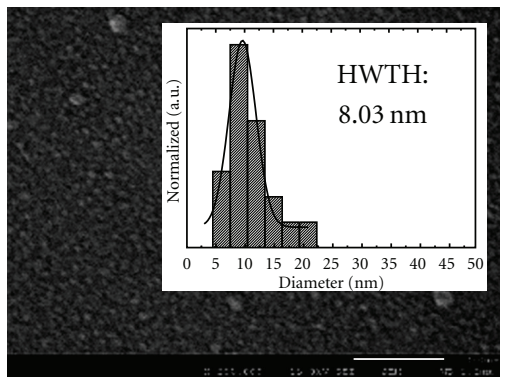

(e)

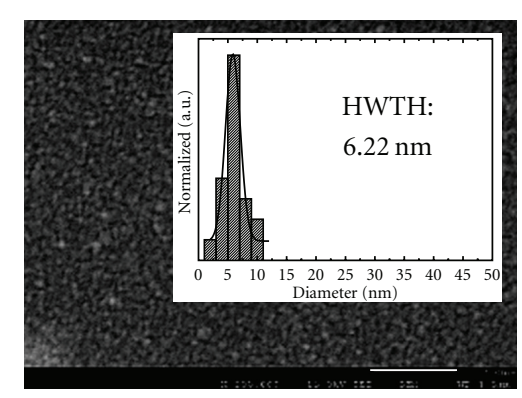

(g)

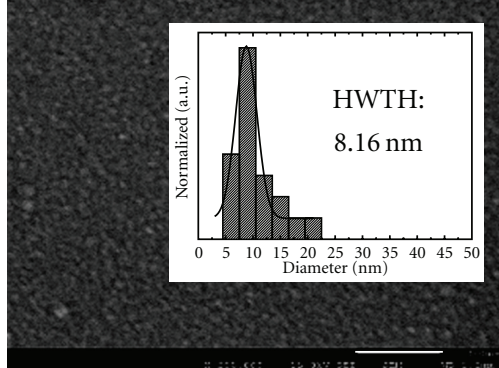

(c)

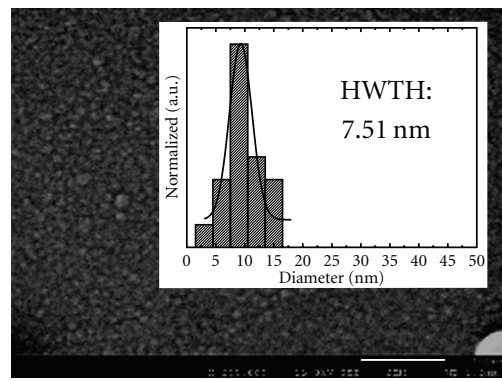

(f)

FIgURE 4: SEM images of the Si films deposited by pulsed laser deposition in $10 \mathrm{~Pa}$ Ne environment gas, where (a), (b), (c), (d), (e), (f), and (g) correspond to various angles of fixed Si wafers: $-80^{\circ},-60^{\circ},-30^{\circ}, 0^{\circ}, 30^{\circ}, 60^{\circ}$, and $80^{\circ}$, respectively.

is narrow and the formed $\mathrm{np}-\mathrm{Si}$ is small. The atomic weight of $\mathrm{He}, \mathrm{Ne}, \mathrm{Ar}$, and $\mathrm{Si}$ is $4.00 \mathrm{u}, 20.17 \mathrm{u}, 39.95 \mathrm{u}$, and $28.09 \mathrm{u}$, respectively. Obviously, atomic weight of $\mathrm{He}$ is the most different from the Si. Thus, for the same angle, the largest and least uniform nanoparticles in size are found in He gas, and Ne induces the smallest and most uniform np-Sis among all the three deposition gases, which is in good agreement with the SEM images.

3.2. The Damping Coefficient versus the Angle. In inertia model [19], drag force is directly composed of mass displacement and is proportional to the square of velocity $(v)$ of the ejected material throughout flight in the inert ambient gas. The equation of motion for the ejected material is

$$
f=-\alpha v^{2},
$$

where $\alpha$ is the damping coefficient.

In this tentative model, the kinetic energy difference between atoms initially ejected and atoms at the end of flight
$(\Delta T)$ is proportional to the cohesive energy of the as-formed nanoparticles $(\Delta G)$

$$
\Delta G=K \Delta T=K \frac{m v_{0}^{2}}{2}\left[1-\exp \left(-\frac{2 \alpha d}{m}\right)\right],
$$

where $v_{0}$ is the initial velocity, $K$ is proportional constant, $m$ is the mass of the ablated particle, and $d$ is the distance from the target to the position that growth of np-Si ends. In our experimental condition, approximation of low ambient gas density and short target-substrate distance can be applied. Therefore, (2) can be rewritten as follows:

$$
\Delta \mathrm{T} \approx v_{0}^{2} \alpha d .
$$

We assume that cohesive energy of per unit volume $\left(\Delta G_{v}\right)$ is a constant, and the cohesive energy for a spherical nanoparticle with average radius $\left(r_{a}\right)$ can be defined as

$$
\Delta G=\frac{4 \pi r_{a}^{3} \Delta G_{V}}{3} .
$$

The Monte Carlo simulation is a convenient method to demonstrate PLD dynamic process [25]. Using this method, 


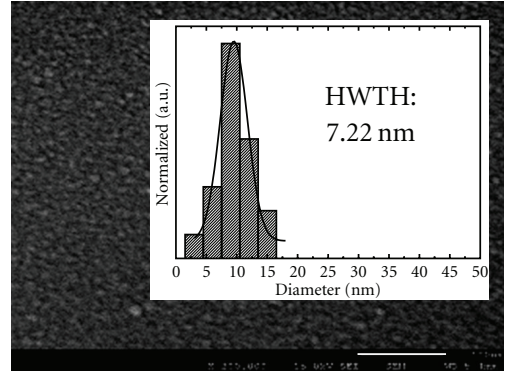

(a)

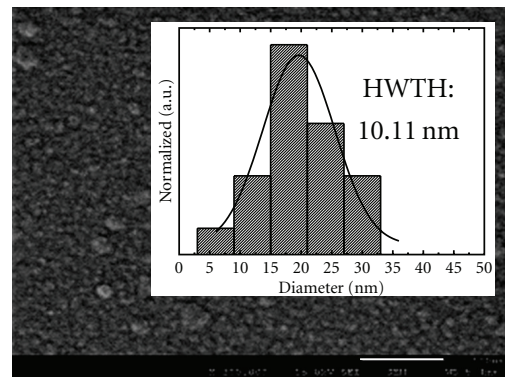

(d)

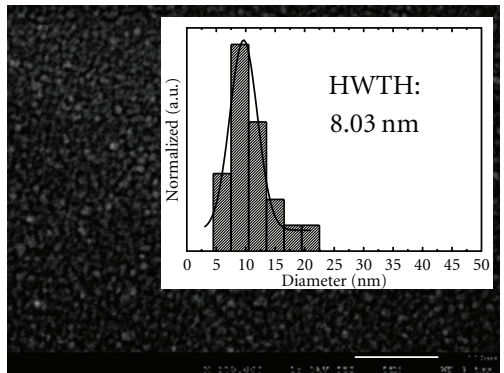

(b)

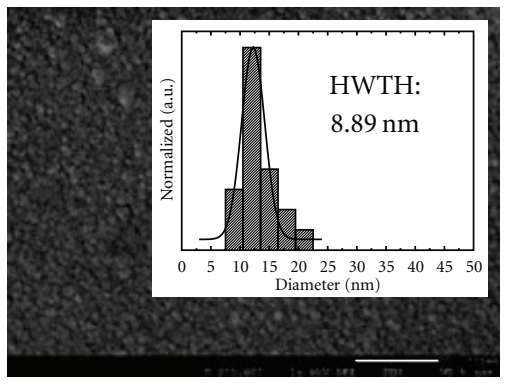

(e)

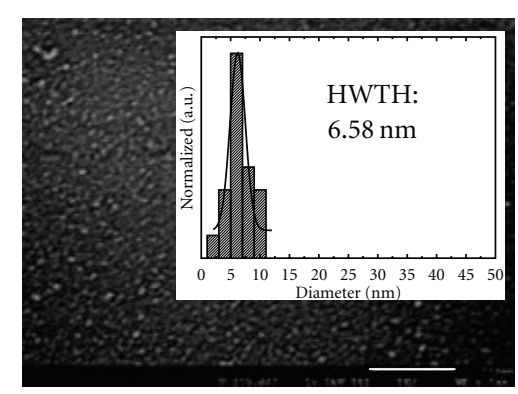

(g)

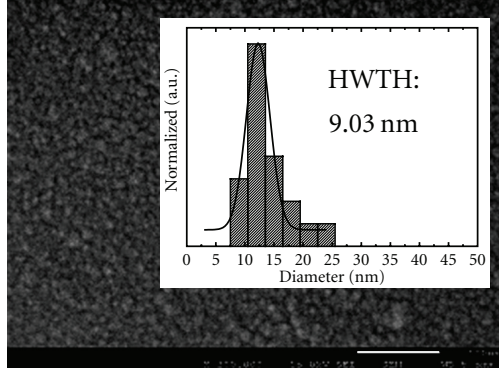

(c)

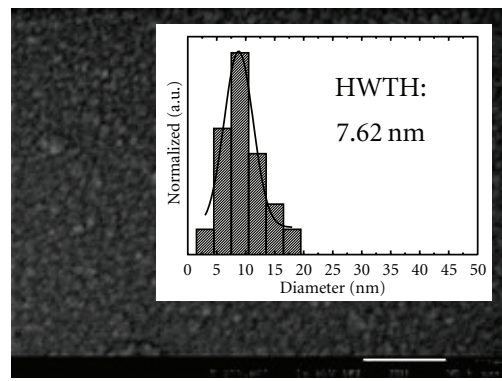

(f)

Figure 5: SEM images of the Si films deposited by pulsed laser deposition in $10 \mathrm{~Pa}$ Ar environment gas, where (a), (b), (c), (d), (e), (f), and (g) correspond to various angle of fixed Si wafers: $-80^{\circ},-60^{\circ},-30^{\circ}, 0^{\circ}, 30^{\circ}, 60^{\circ}$, and $80^{\circ}$, respectively.

we can calculate that the average velocity of ablated particles along plume axis is $v_{0}=1760 \mathrm{~m} / \mathrm{s}$, and the growth position (the mixed region of high density Si vapor and the ambient gas) oscillates continuously between the target and substrate. The maximal positions $(d)$ for $\mathrm{He}, \mathrm{Ar}$, and $\mathrm{Ne}$ are $0.97 \mathrm{~cm}$, $0.71 \mathrm{~cm}$, and $0.60 \mathrm{~cm}$, respectively.

According to the literature [24], the velocity at $\theta$ directions is

$$
v_{\theta}=v_{0} \cos \theta \text {, }
$$

where $\theta$ represents the angle formed by the substrate position and plume axis. Combining (2) and (5), we can get the damping coefficient, defined as

$$
\alpha=\frac{4 \pi r_{a}^{3} \Delta G_{v}}{3 K v_{\theta}^{2} d} .
$$

According to (6) and depending on the average size and velocity of ablated particles at different angles under pure $\mathrm{He}$, $\mathrm{Ne}$, and Ar gases, respectively, the relationship of damping coefficient and the angle is fitted, as shown in Figure 7.
The theoretical simulation demonstrates the bigger the departure angle, the smaller the damping coefficient of ablated particle, and the damping coefficient in Ne gas is largest for the same angle, implying the most effective energy transfer between $\mathrm{Si}$ and ambient atoms.

\section{Conclusions}

In conclusion, angular distribution of damping coefficient of ablated particle under various ambient gases ( $\mathrm{He}, \mathrm{Ar}$ and $\mathrm{Ne}$ ) has been studied. The results show that the average size of $\mathrm{Si}$ nanoparticles decreases with the increase of the departure angle between the film and the plume, and $\mathrm{Ne}$ gas induces the smallest and most uniform Si nanoparticles in size among all the three gases. Further theoretical simulation demonstrates the bigger the departure angle, the smaller the damping coefficient of ablated particle, and the damping coefficient in $\mathrm{Ne}$ gas is largest for the same angle, implying the most effective energy transfer between Si and ambient atoms. This may pave the way for effectively controlling the grain size and 


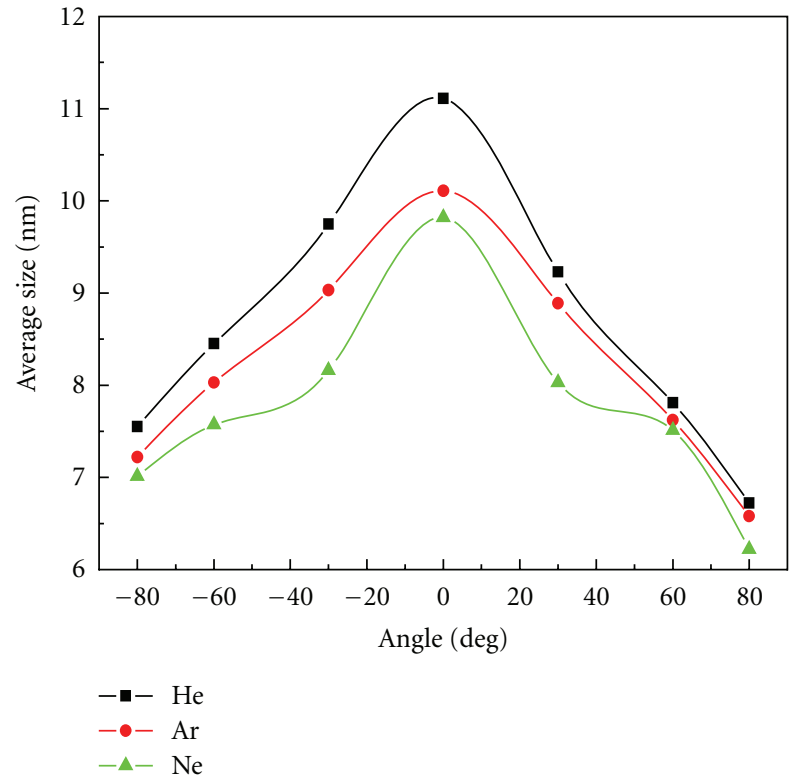

FIGURE 6: The average size versus the angle by pulsed laser deposition in $10 \mathrm{~Pa} \mathrm{He}(-\boldsymbol{-}-), \mathrm{Ar}(-\bullet-)$, and $\mathrm{Ne}(-\mathbf{\Delta}-)$, respectively.

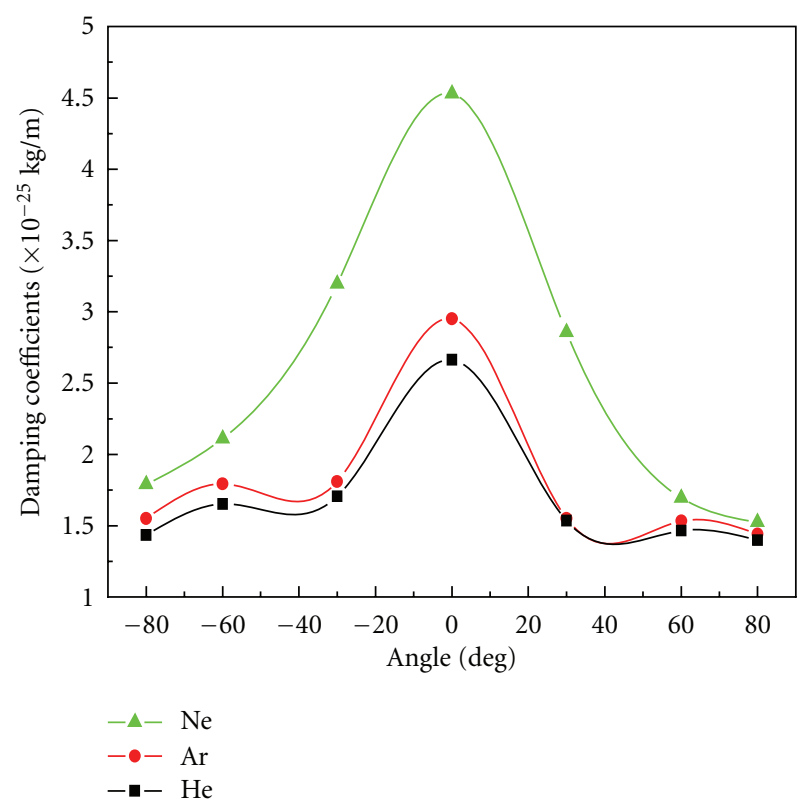

Figure 7: The damping coefficient versus the angle by pulsed laser deposition in $10 \mathrm{~Pa} \mathrm{He}(-\mathbf{-}-), \operatorname{Ar}(-\bullet-)$, and $\mathrm{Ne}(-\mathbf{\Delta}-)$, respectively.

size distribution of nanoparticles of silicon as well as other materials prepared by the pulsed-laser ablation method.

\section{Acknowledgments}

The paper was financially supported by Project supported by the National Basic Research Program of China (Grant no. 2011CB612305), National Science Foundation of China (NSFC) (Grant no. 10774036), the NSF of Hebei Province (E2011201134) and foundation of Hebei University. Support from the Hebei Key Laboratory of Optic-electronic Information \& Materials is also acknowledged.

\section{References}

[1] D. Kovalev, H. Heckler, G. Polisski, and F. Koch, "Optical properties of Si nanocrystals," Physica Status Solidi (B), vol. 215, no. 2, pp. 871-932, 1999.

[2] L. Pavesi, L. Dal Negro, C. Mazzoleni, G. Franzò, and F. Priolo, "Optical gain in silicon nanocrystals," Journal of Physics: Condensed Matter, vol. 4293, no. 162, pp. 440-444, 2001.

[3] B. Goller, S. Polisski, H. Wiggers, and D. Kovalev, "Freestanding spherical silicon nanocrystals: a model system for studying confined excitons," Applied Physics Letters, vol. 97, no. 4, Article ID 041110, 3 pages, 2010.

[4] F. Tian, J. Sun, S. L. Hu, and X. W. Du, "Growth dynamics of nanodiamonds synthesized by pulsed-laser ablation," Journal of Applied Physics, vol. 104, no. 9, Article ID 096102, 3 pages, 2008.

[5] A. S. Mahmood, M. Sivakumar, K. Venkatakrishnan, and B. Tan, "Enhancement in optical absorption of silicon fibrous nanostructure produced using femtosecond laser ablation," Applied Physics Letters, vol. 95, no. 3, Article ID 034107, 3 pages, 2009.

[6] L. Paviesi, "Silicon-based light sources for silicon integrated circuits," Advances in Optical Technologies, vol. 2, no. 6, Article ID 416926, 12 pages, 2008.

[7] S. Lugomer, A. Maksimović, A. Karacs, and A. L. Toth, "Nanostructuring of a silicon surface by laser redeposition of Si vapor," Journal of Applied Physics, vol. 106, no. 11, Article ID 114308, 14 pages, 2009.

[8] S. Ren, Y. Rong, T. I. Kamins, J. S. Harris, and D. A. B. Miller, "Selective epitaxial growth of $\mathrm{Ge} / \mathrm{Si}_{0.15} \mathrm{Ge}_{0.85}$ quantum wells on Si substrate using reduced pressure chemical vapor deposition," Applied Physics Letters, vol. 98, no. 15, Article ID 151108, 3 pages, 2011.

[9] T. F. Deutsch, D. J. Ehrlich, D. D. Rathman, D. J. Silversmith, and R. M. Osgood, "Electrical properties of laser chemically doped silicon," Applied Physics Letters, vol. 39, no. 10, pp. 825$827,1981$.

[10] Y.-H. So, A. Gentle, S. Huang, G. Conibeer, and M. A. Green, "Size dependent optical properties of Si quantum dots in Si-rich nitride/Si3N4 superlattice synthesized by magnetron sputtering," Journal of Applied Physics, vol. 109, no. 6, Article ID 064302, 5 pages, 2011.

[11] H. Y. Chen, H. W. Lin, C. H. Shen, and S. Gwo, "Structure and photoluminescence properties of epitaxially oriented GaN nanorods grown on $\mathrm{Si}(111)$ by plasma-assisted molecularbeam epitaxy," Applied Physics Letters, vol. 89, no. 24, Article ID 243105, 3 pages, 2006.

[12] A. Ishizaka and Y. Shiraki, "Low temperature surface cleaning of silicon and its application to silicon MBE," Journal of the Electrochemical Society, vol. 133, no. 4, pp. 666-671, 1986.

[13] Y. C. Her and C. L. Wu, "Crystallization kinetics of $\mathrm{Cu} / \mathrm{a}$ Si bilayer recording film under thermal and pulsed laser annealing," Journal of Applied Physics, vol. 96, no. 10, article 5563, 3 pages, 2004.

[14] H. Y. Kwong, M. H. Wong, C. W. Leung, Y. W. Wong, and K. H. Wong, "Formation of core/shell structured cobalt/carbon nanoparticles by pulsed laser ablation in toluene," Journal of Applied Physics, vol. 108, no. 3, Article ID 034304, 5 pages, 2010. 
[15] N. G. Semaltianos, S. Logothetidis, W. Perrie et al., "Silicon nanoparticles generated by femtosecond laser ablation in a liquid environment," Journal of Nanoparticle Research, vol. 12, no. 2, pp. 573-580, 2009.

[16] Y. C. Peng, G. S. Fu, W. Yu, S. Q. Li, and Y. L. Wang, "Crystallization of amorphous Si films by pulsed laser annealing and their structural characteristics," Semiconductor Science and Technology, vol. 19, no. 6, pp. 759-763, 2004.

[17] D. Riabinina, E. Irissou, B. Le Drogoff, M. Chaker, and D. Guay, "Influence of pressure on the Pt nanoparticle growth modes during pulsed laser ablation," Journal of Applied Physics, vol. 108, no. 3, Article ID 034322, 6 pages, 2010.

[18] Y. L. Wang, Z. C. Deng, G. S. Fu, Y. Zhou, L. Z. Chu, and Y. C. Peng, "The average size of Si nanoparticles prepared by pulsed laser ablation in the gas mixture of $\mathrm{He} / \mathrm{Ar}, \mathrm{Ne} / \mathrm{Ar}$ or $\mathrm{He} / \mathrm{Ne}$," Thin Solid Films, vol. 515, no. 4, pp. 1897-1900, 2006.

[19] T. Yoshida, S. Takeyama, Y. Yamada, and K. Mutoh, "Nanometer-sized silicon crystallites prepared by excimer laser ablation in constant pressure inert gas," Applied Physics Letters, vol. 68, no. 13, pp. 1772-1774, 1996.

[20] G. S. Fu, Y. L. Wang, L. Z. Chu et al., "The size distribution of Si nanoparticles prepared by pulsed-laser ablation in pure $\mathrm{He}$, Ar or Ne gas," Europhysics Letters, vol. 69, no. 5, pp. 758-762, 2005.

[21] Y. Wang, W. Xu, Y. Zhou, L. Chu, Y. Hou, and G. Fu, "Influence of laser beam on transport dynamics of Si nanoparticles by laser ablation,” vol. 6722, no. 4, pp. H1-H7.

[22] E. Werwa, A. A. Seraphin, L. A. Chiu, C. Zhou, and K. D. Kolenbrander, "Synthesis and processing of silicon nanocrystallites using a pulsed laser ablation supersonic expansion method," Applied Physics Letters, vol. 64, no. 14, pp. 18211823, 1994.

[23] L. V. Zhigilei and B. J. Garrison, "Velocity distributions of molecules ejected in laser ablation," Applied Physics Letters, vol. 71, no. 4, pp. 551-553, 1997.

[24] L. Torrisi, G. Ciavola, S. Gammino et al., "Metallic etching by high power Nd:yttrium-aluminum-garnet pulsed laser irradiation," Review of Scientific Instruments, vol. 71, no. 11, pp. 4330-4334, 2000.

[25] Y. L. Wang, L. Z. Chu, Y. L. Li, and G. S. Fu, "Dynamical transportation of Si particles produced by pulsed laser ablation in the mixture of two inert gases," Micro \& Nano Letters, vol. 4, no. 1, pp. 39-43, 2009. 

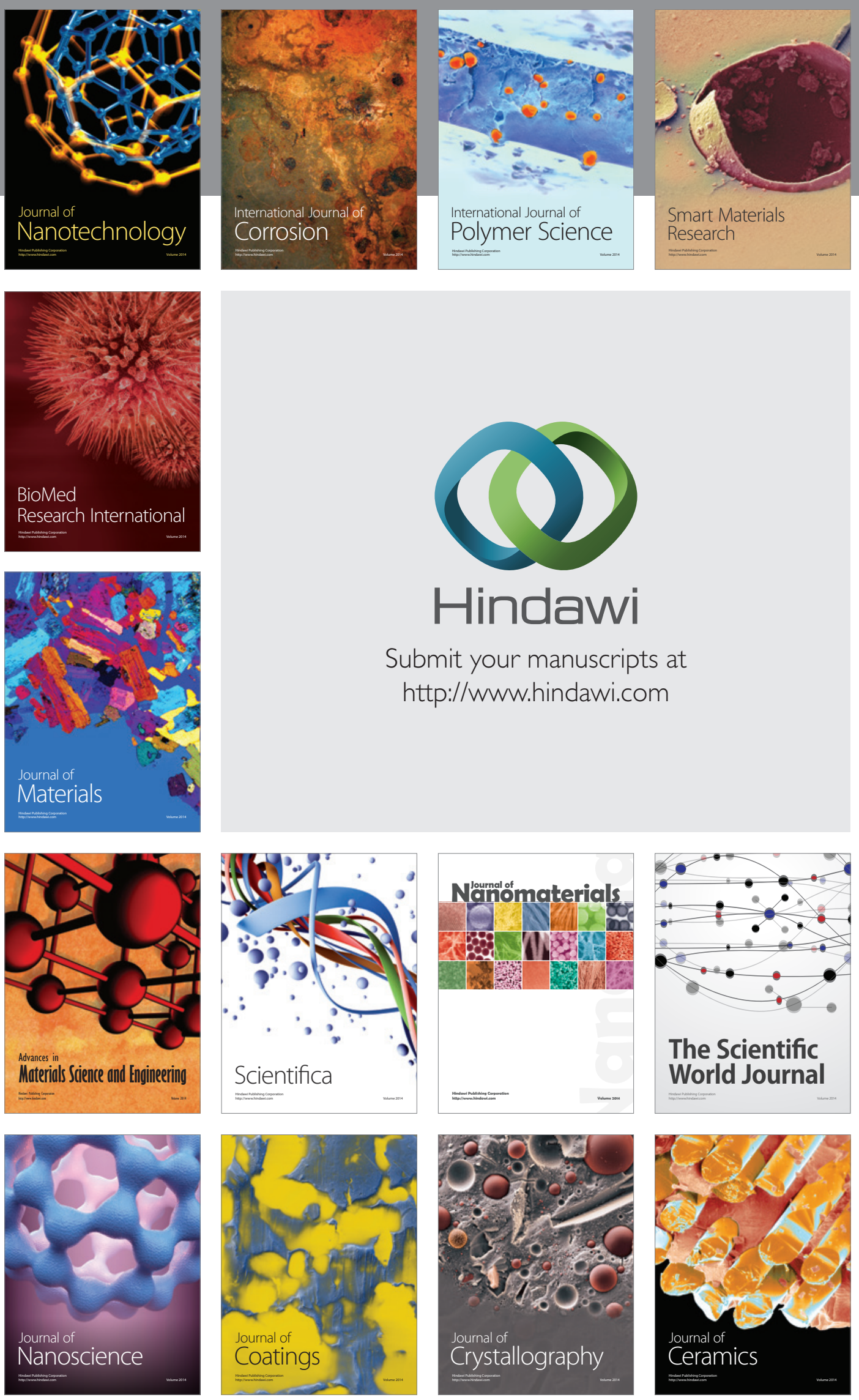

The Scientific World Journal

Submit your manuscripts at

http://www.hindawi.com

\section{World Journal}

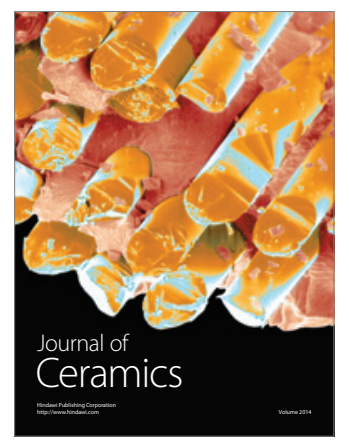


